\title{
CXCR4 nuclear localization follows binding of its ligand SDF-1 and occurs in metastatic but not primary renal cell carcinoma
}

\author{
LINHUI WANG ${ }^{*}$, ZHIXIANG WANG* ${ }^{*}$ BO YANG, QING YANG, LIANG WANG and YINGHAO SUN \\ The Department of Urology, Changhai Hospital, Second Military Medical University, Shanghai, P.R. China
}

Received April 22, 2009; Accepted June 1, 2009

DOI: $10.3892 /$ or_00000572

\begin{abstract}
Renal cell carcinoma (RCC) shows organ-specific metastasis. This may be attributed to the fact that the CXCR4 $\mathrm{G}$ protein coupled receptor on RCC cells mediates chemoattraction toward stromal-derived factor (SDF)-1 secreted by target organs. SDF-1 binding to CXCR4 initiates many internal signaling cascades as well as internalization of the receptor complex. Here we show that SDF-1 binding localized CXCR4 to the nucleus in A-498 renal carcinoma cells over a $24 \mathrm{~h}$ period. CXCR4 nuclear localization in A-498 cells associated with increased Matrigel matrix invasion, a metastatic trait. Comparing histological sections of primary and metastatic human RCC, we found that CXCR4 localized to the nucleus only in metastatic RCC lesions. In conclusion, SDF-1 binding of CXCR4 not only induces immediate signaling via signaling cascades, but also a slower response via nuclear localization of the receptor complex. CXCR4 nuclear localization may be responsible for certain metastatic changes in cancer cells and can be used to distinguish metastatic RCC cells. These findings may be applied to the search for ways to inhibit RCC, as well
\end{abstract}

Correspondence to: Dr Yinghao Sun, The Department of Urology, Changhai Hospital, Second Military Medical University, 200433 Shanghai, P.R. China

E-mail: sunyhchanghai@yahoo.cn

${ }^{*}$ Contributed equally

Abbreviations: CT, computed tomography; ECL, enhanced chemiluminescence; EGFP, enhanced green fluorescent protein; EGFR, epidermal growth factor receptor; EMEM, ATCC-formulated Eagle's Minimum Essential Medium; FITC, fluorescein isothiocyanate; G418, geneticin; H\&E, Hematoxylin and eosin staining; HIV, human immunodeficiency virus; HSC, hematopoietic stem cell; Hsc73, $73 \mathrm{kDa}$ heat shock cognate protein; PI, propidium iodide; PBS, phosphate-buffered saline; RCC, renal cell carcinoma; SDF-1, stromal cell-derived factor-1; SDS-PAGE, sodium dodecyl sulfate polyacrylamide gel electrophoresis

Key words: CXCR4 receptor, CXCL12 chemokine, stromal cellderived factor 1, renal cell carcinoma, nuclear localization signals, cancer as to many other questions that involve CXCR4 such as normal hematopoiesis, tissue regeneration, stem cell research and HIV infection.

\section{Introduction}

One of the main causes of mortality from cancer is metastasis to vital organs such as the lungs or bone marrow. The metastasis of renal cell carcinoma in particular is a troublesome clinical problem with high morbidity and mortality. However, out of thousands of tumor cells, only a few escape the primary tumor by metastasis to colonize target organs. The CXCR4/ SDF-1 pathway has been identified as a main mechanism involved in organ-specific metastasis of cancer (1-3). CXCR4 appears to be the major chemokine receptor expressed on cancer cells (4-7) and renal cell carcinoma cells express a high level of CXCR4 compared to normal renal tissue $(8,9)$. The ligand for CXCR4 is $\alpha$-chemokine stromal-derived factor (SDF)-1 (also named CXCL12) and is expressed at high levels by the organs to which RCC metastasizes (1). Thus, treatment of RCC may benefit from a deeper understanding of the CXCR4/SDF-1 signaling pathway. In the future, intractable metastatic cancers such as renal cell carcinoma may be treated by attacking the CXCR4/SDF-1 pathway.

Understanding the CXCR4 cytokine receptor is relevant to many branches of biology and medicine beyond cancer. Unlike the other members of the cytokine receptor family that promiscuously bind a wide range of cytokines, CXCR4 is very specific for its ligand, SDF-1. This pairing specificity may be necessary due to the role of the CXCR4/SDF-1 signaling as a chemoattractant pathway for stem cells to home to target tissues (1). Hematopoietic stem cell (HSC) transplants may be given intravenously, yet the cells spontaneously colonize the bone marrow; this clinically convenient fact may be explained by the existence of the CXCR4/SDF-1 system in which CXCR4-bearing stem cells are attracted to the SDF-1-secreting bone marrow. The CXCR4/SDF-1 attractant system seems to be used by all stem cells, not only HSCs and therefore is of great interest to stem cell therapy practitioners. CXCR4 is also of interest to human immunodeficiency virus (HIV) researchers because the X4 HIV strain docks with CXCR4 and CD4 via the HIV protein gp120 to gain entry into lymphocytes (10).

The chemokine receptor CXCR4 belongs to the large superfamily of $\mathrm{G}$ protein-coupled receptors $(11,12)$. SDF-1 binding to CXCR4 leads to the activation of multiple $\mathrm{G}$ protein-dependent signaling pathways, resulting in diverse 
biological outcomes such as migration, adhesion and transcriptional activation (13). Phosphorylation of CXCR4 tyrosine residues has also been observed following SDF-1 stimulation (14). The chemokine SDF-1 triggers CXCR4 receptor dimerization and activates the JAK/STAT pathway (14).

Binding of SDF-1 to CXCR4 also results in internalization of the receptor complex. The tyrosine phosphorylation induced by SDF-1 may be a way of promoting ligand-independent internalization of CXCR4 (15). In unstimulated cells CXCR4 is found both on the cell surface and internally, presumably recycling to the cell surface through endosomes $(16,17)$. In cells stimulated with SDF-1 there is internalization of most of the surface CXCR4 to endosomes and lysosomes (18). Internalization of CXCR4 seems to be independent of $\mathrm{G}$ protein signaling because pertussis toxin does not inhibit the internalization process (17).

Internalization of CXCR4 after SDF-1 treatment involves interaction with the proteins ferritin (19), 73-kDa heat shock cognate protein (Hsc73) (20), plectin (10) and Myosin IIA (21). A recent study found several proteins, including ferritin (19) and Hsc73 (20), in combination with CXCR4 after internalization of CXCR4. The ferritin heavy chain and Hsc73 are not only found as CXCR4 binding proteins but also regulate the receptor endocytosis and the receptor-mediated chemotaxis $(19,20)$. It has also been reported that $B$-arrestin may regulate the internalization of CXCR4 (22). Plectin is required for CXCR4 internalization, is found in intracellular vesicles with CXCR4 and is required for ERK1/2 signaling (10). Myosin IIA is required for SDF-1-induced endocytosis of CXCR4 (21).

Other receptors, such as the epidermal growth factor receptor (23), are known to localize to the nucleus after ligand binding. Therefore, we hypothesized that CXCR4 may also localize to the nucleus following ligand binding. This study extends previous observations about the fate of CXCR4 internalization following SDF-1 stimulation and explores its consequences with regard to RCC.

\section{Materials and methods}

Cell culture and transfection. A-498 (ATCC, Manassas, VA, USA), a human renal cell carcinoma cell line, was grown at $37^{\circ} \mathrm{C}$ in a humidified atmosphere of $95 \%$ air and $5 \% \mathrm{CO}_{2}$ in ATCC-formulated Eagle's Minimum Essential Medium (EMEM, Cat. No. 30-2003, ATCC) supplemented with $10 \%$ fetal bovine serum.

Because A-498 cells have low intrinsic CXCR4 expression (24), the plasmid pcDNA3.1-CXCR4 was constructed to overexpress CXCR4. Briefly, a cDNA of human CXCR4 from a cDNA library of A-498 cells was amplified by polymerase chain reaction (PCR) using a pair of specific primers: 5'-ATC GTGAAGCTTATGGAGGGGATCAGTATATACACTTCA G-3', and 5'-TGCATACTCGAGTTAGCTGGAGTGAAAA CTTGAAGACTCA-3'. The amplified fragment, containing the human CXCR4 open reading frame with a 5' HindIII site and 3' XholI site, was inserted into the BamHI (Takara Biomedicals, Dalian, China) and XholI (Takara Biomedicals) site of the pcDNA3.1 vector (Invitrogen, Carlsbad, CA, USA).

We also constructed the mammalian expression vector pEGFP-CXCR4 carrying CXCR4 fused to enhanced green fluorescent protein (EGFP). Briefly, A human CXCR4 cDNA fragment was amplified from a cDNA library of A-498 cells using a pair of specific primers: CXCR4E-UP, 5'-AGCTTA ATGGAGGGGATCAGTATATACACTTCAG-3', and CXCR4X-DN, 5'-CAGATCTGAGCTGGAGTGAAAACTT GAAGACTCAGA-3'. This fragment contained the human CXCR4 open reading frame with a $5^{\prime}$ HindIII site and a $3^{\prime}$ $B g l$ II site. This fragment was ligated upstream of the GFP gene into the HindIII (Takara Biomedicals) and BamHI site of pEGFP-N1 (Cat. No. 6085-1, Clontech, Palo Alto, CA, USA).

A-498 cells were transfected with plasmids using Lipofectamine 2000 reagent (Invitrogen) according to the manufacturer's instructions. Positive clones with stable expression were selected with $500 \mu \mathrm{g} / \mathrm{ml}$ geneticin (G418; Gibco-BRL, Grand Island, NY, USA); selection started $48 \mathrm{~h}$ after transfection. Individual G418-resistant cells were observed after 8 days of G418 selection and G418-resistant colonies were observed after 28 days of G418 selection. The integrity and fidelity of the cloned CXCR4 cDNA and EGFPCXCR4 sequences were confirmed by DNA sequencing on an ABI DNA sequencer (ABI, Foster City, CA, USA).

Confocal microscopy. A-498 cell clones stably expressing plasmids pcDNA3.1-CXCR4 or pEGFP-CXCR4 were grown on coverslips ( $4 \times 10^{4}$ cells/coverslip) for microscopy studies. The cells were treated with either carrier buffer or $200 \mathrm{ng} / \mathrm{ml}$ SDF-1 (R\&D Systems, Minneapolis, MN, USA) for various time intervals and then observed. For observation, the cells were fixed with methanol, washed with phosphate-buffered saline (PBS) and incubated for $30 \mathrm{~min}$ with an antibody mixture containing a rabbit polyclonal anti-CXCR4 antibody (AB1846, Chemicon, Temecula, CA, USA). Cells were then washed and incubated for 30 min with a secondary antibody mixture containing a FITC-conjugated anti-rabbit antibody (Molecular Probes, Eugene, OR, USA). After immunostaining, the cells were counterstained with propidium iodide (PI) to visualize nuclei. Confocal microscopy was performed on an LSM-510 laser scanning microscope (Carl Zeiss, Thornwood, NY, USA) with a x63, 1.3 numerical aperture oil immersion lens using dual excitation (488 nm for EGFP or FITC, $490 \mathrm{~nm}$ for PI) and emission (515-540 nm for EGFP or FITC, 580$620 \mathrm{~nm}$ for PI) filter sets. All digital images were captured at the same settings to allow direct quantitative comparison of staining patterns. Final images were processed using Adobe Photoshop software.

Western blotting of CXCR4 proteins extracted from nuclei. A-498 cells $\left(5 \times 10^{6} / \mathrm{ml}\right)$ stably expressing pcDNA3.1-CXCR4 were treated with either carrier buffer (control) or $200 \mathrm{ng} / \mathrm{ml}$ SDF-1 for the indicated time intervals. After washing the cells free of medium with ice-cold phosphate-buffered saline three times, nuclear and cytoplasmic protein fractions were extracted with Nuclear and Cytoplasmic Extraction Reagents (NE-PER, Pierce, Rockford, IL, USA) according to the manufacturer's instructions. The protein concentration was determined by BCA assay (Pierce) and $20 \mathrm{mg}$ protein/lane was separated by SDS-PAGE on $12 \%$ gradient gels (Novex, Invitrogen). Western blots for CXCR4 were visualized with ECL detection reagents (Amersham, Uppsala, Sweden). Blots were probed 
for CXCR4 (anti-CXCR4 antibody) and, as a positive control for a nuclear protein, SP-1 (anti-SP-1 antibody; Santa Cruz, Santa Cruz, CA, USA). When reprobing was necessary, the blots were stripped using Restore Western Blotting Stripping Reagent (Pierce) and then reprobed.

In vitro invasion assays. A-498 cells were assayed for their invasion ability using Biocoat Matrigel invasion chambers (BD Biosciences, Franklin Lakes, NJ, USA) as described in the manufacturer's protocol with modifications. Briefly, 24-well Matrigel invasion chambers were rehydrated with EMEM containing $0.2 \%$ bovine serum albumin for $2 \mathrm{~h}$ at room temperature. Then, A-498 cells transfected with either pcDNA3.1-CXCR4 or control vector (pcDNA3.1) were seeded into the upper well of invasion chambers $\left(5 \times 10^{5}\right.$ cells $/ 0.4 \mathrm{ml}$ of medium containing $0.2 \%$ bovine serum albumin). The lower chambers were filled with $0.4 \mathrm{ml}$ of medium containing $10 \%$ fetal bovine serum and $200 \mathrm{ng} / \mathrm{ml} \mathrm{SDF}-1$. The cells were allowed to invade Matrigel matrices for $12 \mathrm{~h}$ at $37^{\circ} \mathrm{C}$ in a $\mathrm{CO}_{2}$ incubator. To count cells that migrated onto the lower surface of the membrane, the cells on the upper side of the membrane were scraped off with a cotton swipe and the inserts containing the membrane were stained with $0.1 \%$ crystal violet. The crystal violet was removed by 3 washes with PBS, and then the remaining crystal violet that had stained the migrated cells was eluted with 1 wash of $33 \%$ acetic acid. The $\mathrm{OD}_{540}$ of the eluted crystal violet was determined as a measure of migrated cells.

Renal cell carcinoma tissue specimens. To examine the distribution of CXCR4 in renal cancer cells, we obtained surgical resection samples of human renal cell carcinomas (RCC) from the sample bank in the Department of Pathology, Changhai Hospital, Shanghai. The samples originated from 3 patients with metastatic RCC, who provided written informed consent to use their specimens. The specimens were cryopreserved, fixed in formalin and sectioned (4- $\mu \mathrm{m}$-thick). The samples were examined by a pathologist and categorized as primary RCC lesions or metastatic RCC lesions, based on morphology (H\&E), immunohistochemistry and patient CT scans for metastasis. The study design was approved by the Changhai Hospital Ethics Committee.

Immunofluorescent detection of CXCR4 in RCC. CXCR4 intracellular distribution in both primary and metastatic RCC was studied by immunofluorescent confocal microscopy. Tissue sections were incubated for $1 \mathrm{~h}$ with polyclonal rabbit anti-human CXCR4 antibody (AB1846; Chemicon), followed by incubation for $1 \mathrm{~h}$ with FITC-conjugated goat anti-rabbit secondary antibody (1: 250). The sections were counterstained with PI to visualize nuclei and observed by confocal microscopy as above.

Statistical analysis. The Mann-Whitney U test was used to compare variables between SDF-1-treated and untreated samples. The Kruskal-Wallis test was used to test the difference among 3 groups. When a significant difference between groups was apparent, multiple comparisons of means were performed using the Bonferroni procedure with type-I error adjustment. Data are presented as means \pm SD. All statistical assessments were two-sided and evaluated at the 0.05 level of significant difference. Statistical analyses were performed using SPSS 15.0 statistics software (SPSS, Chicago, IL, USA).

\section{Results}

CXCR4 after SDF-1 stimulation. To follow the fate of CXCR4 after SDF-1 stimulation, A-498 cells expressing CXCR4 from the pcDNA3.1-CXCR4 plasmid were grown on coverslips for $24 \mathrm{~h}$ with either carrier buffer (control) or $200 \mathrm{ng} / \mathrm{ml}$ SDF-1. CXCR4 was visualized on a confocal microscope with immunofluorescent staining for CXCR4 and PI staining for nuclei (Fig. 1). When the cells were stimulated with SDF-1, there was strong CXCR4 staining in distinct vesicle-like spots (probably endo/lysosomal compartments) in the cytoplasm at $0 \mathrm{~h}$ (Fig. 1A) and $6 \mathrm{~h}$ (data not shown), then staining both in spots and diffuse throughout the cytoplasm and nucleus at $12 \mathrm{~h}$ (Fig. 1B) and finally diffuse cytoplasmic and nuclear staining without any spots at $24 \mathrm{~h}$ (Fig. 1C). In contrast, control cells only showed CXCR4 staining in distinct spots at all time points (data not shown). To confirm the data from immunohistochemical staining for CXCR4, A-498 cells were transfected with CXCR4 fused to EGFP. The same results were seen for SDF-1 stimulation (Fig. 1D) and controls (not shown).

To verify that the nuclear and cytoplasmic distribution of CXCR4 was not a microscopy artifact, Western blots of the nuclear and cytoplasmic fractions were tested for CXCR4. CXCR4 was present in both nuclear and cytoplasmic fractions in SDF-1 stimulated cells (Fig. 2). In control cells, CXCR4 was mostly found in the cytoplasmic fraction with a small amount in the nuclear fraction (Fig. 2); although all due care was taken, the fractionation method is known to have a small amount of cross-contamination of cytoplasmic and nuclear proteins even under optimum conditions.

CXCR4 nuclear localization is associated with increased invasiveness. To explore whether internalization of CXCR4 to the nucleus had a functional consequence, we assayed the ability of A-498 cells to invade a Matrigel-coated membrane (Fig. 3), which is similar to extravasation through an extracellular matrix. Cells were added to the upper chamber of a Biocoat Matrigel invasion chamber and 0 or $200 \mathrm{ng} / \mathrm{ml} \mathrm{SDF-1}$ was added to the lower chamber as a chemoattractant. The cells were allowed $12 \mathrm{~h}$ to migrate across the membrane.

A-498 cells natively express a low amount of CXCR4. When control A-498 cells carrying an empty pcDNA3.1 plasmid were treated with SDF-1 and immediately placed in the upper chamber, some cells crossed the membrane without an SDF-1 gradient (0 $\mathrm{ng} / \mathrm{ml} \mathrm{SDF-1} \mathrm{in} \mathrm{the} \mathrm{lower} \mathrm{chamber)}$ whereas significantly more cells crossed the membrane when an SDF-1 gradient was present (200 ng/ml SDF-1 in the lower chamber).

When A-498 cells carrying an empty pcDNA3.1 plasmid were pretreated with SDF-1 for $24 \mathrm{~h}$ (long enough for CXCR4 to redistribute to the nucleus) and then placed in the upper chamber, some cells crossed the membrane without an SDF-1 gradient whereas significantly more cells crossed the membrane when and SDF-1 gradient was present $(200 \mathrm{ng} / \mathrm{ml}$ SDF-1 in the lower chamber). Both with and without an SDF-1 
A

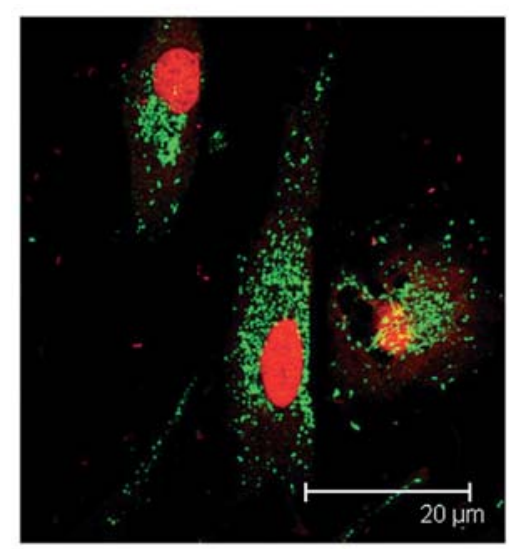

C

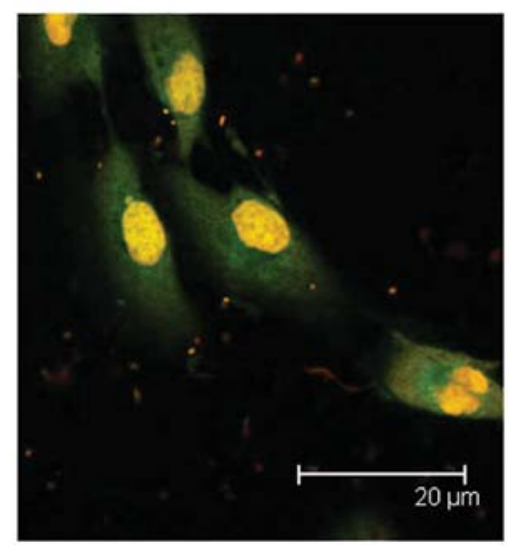

B
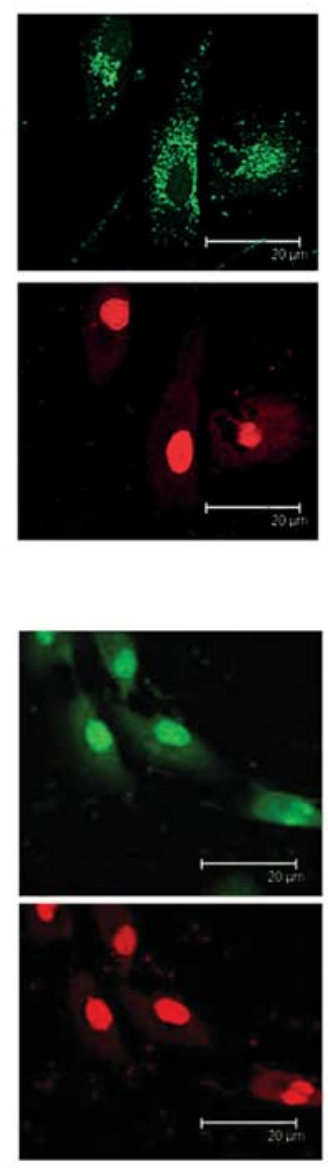

D
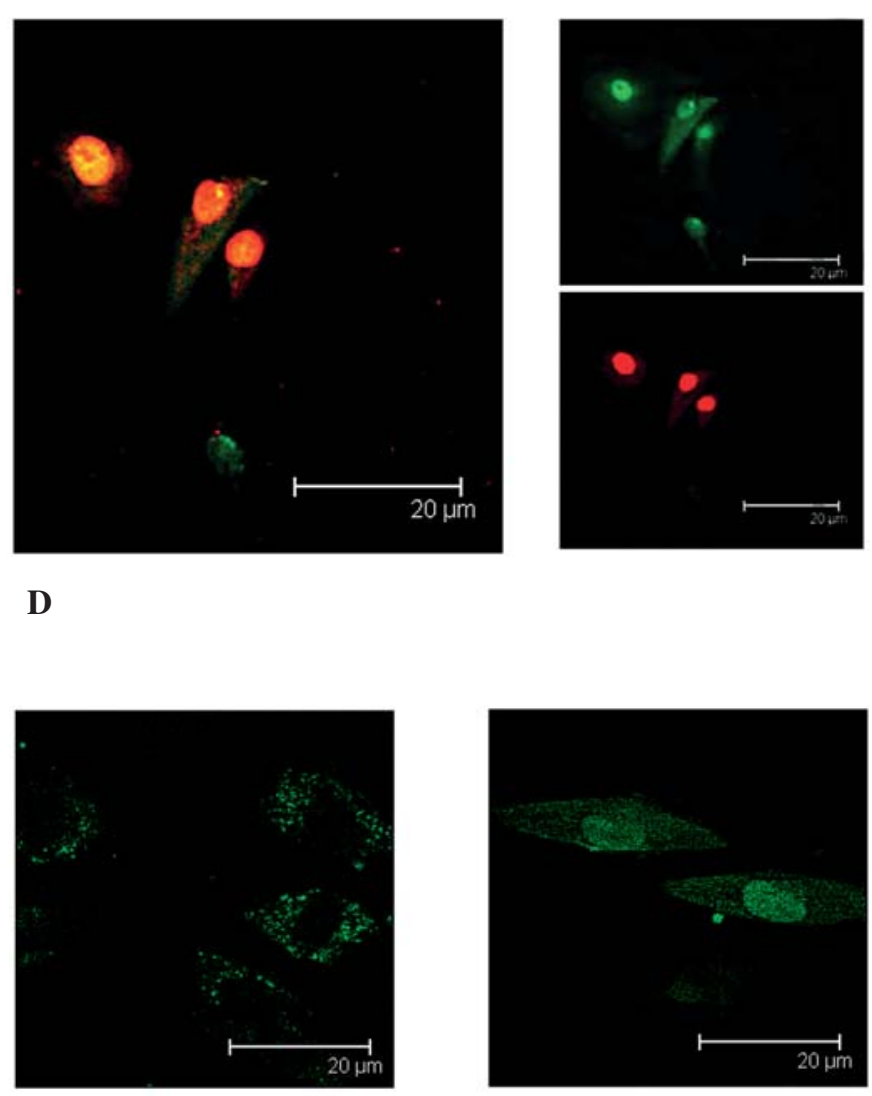

Figure 1. Nuclear localization of CXCR4 after SDF-1 stimulation in vitro. (A-C) A-498 cells overexpressing CXCR4 were stimulated with 200 ng/ml SDF-1 over $24 \mathrm{~h}$ and visualized at 0,6, 12 and $24 \mathrm{~h}$ by immunofluorescence for CXCR4 (green, FITC) and nuclei (red, propidium iodide) on a confocal microscope, with costaining visible in the fused images (yellow). At $0 \mathrm{~h}$ (A) CXCR4 was seen in cytoplasmic vesicles; the same pattern was seen at $6 \mathrm{~h}$ (not shown) and in control cells (no SDF-1 treatment) at all time points (not shown). At $12 \mathrm{~h}$ (B) there was CXCR4 vesicular staining as well as diffuse nuclear and cytoplasmic staining. At $24 \mathrm{~h}$ (C) CXCR4 was only visible as diffuse nuclear and cytoplasmic staining. A repeat of the experiment with EGFP-tagged CXCR4 (green) gave the same results; A-498 cells expressing EGFP-CXCR4 after $24 \mathrm{~h}$ SDF-1 stimulation are shown (D).

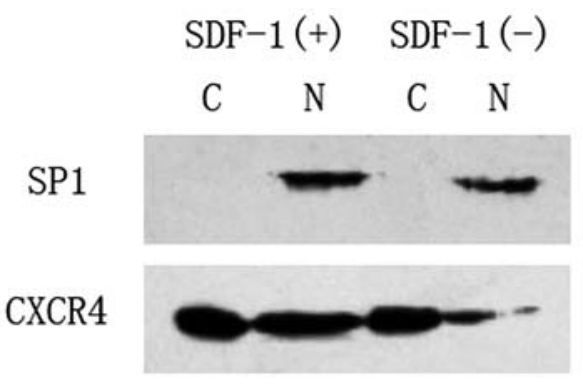

Figure 2. Western blots for CXCR4 in nuclear and cytoplasmic fractions of A-498 cells. A-498 cells overexpressing CXCR4 were treated with carrier buffer (control) or SDF-1 $(200 \mathrm{ng} / \mathrm{ml})$. Cellular proteins were separated into nuclear $(\mathrm{N})$ and cytoplasmic (C) fractions. Western blots were performed for SP-1 (a nuclear fraction protein) and CXCR4.

gradient, SDF-1 pretreated A-498 cells showed significantly higher invasion of the lower chamber than the control A-498 cells. This suggests that the presence of SDF-1-stimulated CXCR4 in the nucleus caused and increase in A-498 invasive migration, a hallmark of metastatic cancer cells.

We further repeated this experiment with A-498 cells overexpressing CXCR4 from the pcDNA3.1-CXCR4 plasmid.
The A-498 pcDNA3.1-CXCR4 cells were treated with SDF-1 and immediately placed in the upper chamber; some cells crossed the membrane without an SDF-1 gradient whereas significantly more cells crossed the membrane when and SDF-1 gradient was present. The invasive migration was less than the SDF-1 pretreated A-498 cells, but still showed significantly higher invasion of the lower chamber than the control A-498 cells.

In summary, short-term treatment (controls) with SDF-1 led to some transmigration of cells and the presence of an SDF-1 chemoattractant gradient increased the transmigration of A-498 cells. Increasing the abundance of CXCR4 by using an overexpression plasmid also increased the responsiveness of A-498 cells to a short-term treatment with SDF-1. Finally, the results of the experiments suggest that translocation of CXCR4 to the nucleus following $24 \mathrm{~h} \mathrm{SDF}-1$ exposure could significantly increase the invasive migratory ability of A-498 cells. The SDF-1/CXCR4 interaction seemed to stimulate invasive migration of A-498 cells in a dose-dependent manner; invasive migration was increased after either increasing the amount of CXCR4 by overexpression or increasing the amount of SDF-1 stimulation by providing a chemoattractant SDF-1 gradient. Further, the stimulation of Matrigel invasion was 


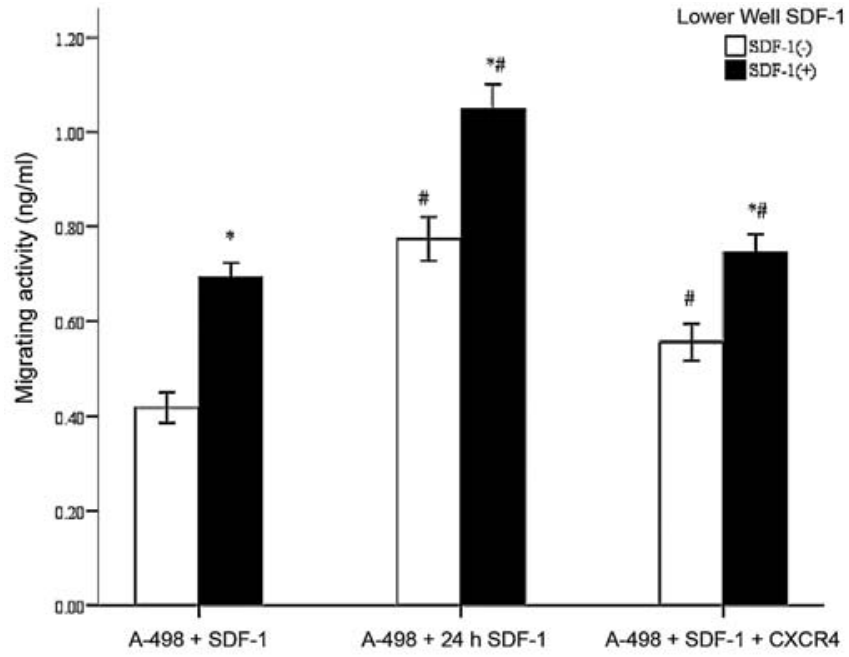

Figure 3. Matrigel invasion assay. A Matrigel invasion assay was performed to see if nuclear localization of CXCR4 (caused by $24 \mathrm{~h}$ pretreatment with SDF-1) affected invasive ability. The following groups were tested. A-498 + SDF-1: A-498 cells transfected with pcDNA3.1 (control) were treated with $200 \mathrm{ng} / \mathrm{ml} \mathrm{SDF}-1$ and immediately placed into Biocoat Matrigel invasion chambers for $12 \mathrm{~h}$. A-498 + 24 h SDF-1: A-498 cells transfected with pcDNA3.1 (control) were pretreated with $200 \mathrm{ng} / \mathrm{ml} \mathrm{SDF}-1$ for $24 \mathrm{~h}$ to allow time for nuclear localization of CXCR4 and then placed into Biocoat Matrigel invasion chambers for $12 \mathrm{~h}$. A-498 + SDF-1 + CXCR4: A-498 cells overexpressing CXCR4 were treated with $200 \mathrm{ng} / \mathrm{ml} \mathrm{SDF}-1$ and immediately placed into Biocoat Matrigel invasion chambers for $12 \mathrm{~h}$. Each group was placed into Biocoat Matrigel invasion chambers with either 0 or $200 \mathrm{ng} / \mathrm{ml} \mathrm{SDF}-1$ in the lower chamber as chemoattractant. Migrating activity is expressed in $\mathrm{ng} / \mathrm{ml}$ crystal violet. Data are presented as mean migrating activities; bars, SD $(n=3)$. *Significant difference from paired white bar (control, $0 \mathrm{ng} / \mathrm{ml}$ SDF-1), $\mathrm{P}<0.05$; "Significant difference from control group (A-498 + SDF-1), $\mathrm{P}<0.05$.

maximized when sufficient time was allowed for translocation of CXCR4 to the nucleus (24 h SDF-1 pretreatment).

Nuclear localization of CXCR4 was found in metastatic but not primary renal cancer lesions. Renal cell carcinoma is characterized by organ-specific metastases and CXCR4 is expressed on circulating cytokeratin positive RCC cells from patients with metastatic RCC (11). The chemokine SDF-1 and its receptor CXCR4 have been suggested to regulate organ- specific metastasis in various cancers. Therefore, we hypothesized that nuclear localization of CXCR4 may promote metastasis of cancers, such as renal cell carcinoma. To evaluate this hypothesis we compared the CXCR4 localization in samples of primary and metastatic RCC lesions.

Tissue sections from primary or metastatic RCC lesions were stained for CXCR4 (Fig. 4). Primary lesions had diffuse CXCR4 staining in the cytoplasm, but not the nucleus (Fig. 4A), whereas metastatic lesions had diffuse CXCR4 staining in both the cytoplasm and nucleus (Fig. 4B and C).

\section{Discussion}

Our results in conjunction with the results of previous observations reveal a more detailed picture of CXCR4 receptor internalization. In unstimulated cells, CXCR4 is found on the cell surface, probably as dimers associated with lipid rafts $(25,26)$. CXCR4 dimerization and association with the lipid rafts may be necessary for function (25). Whereas some
A
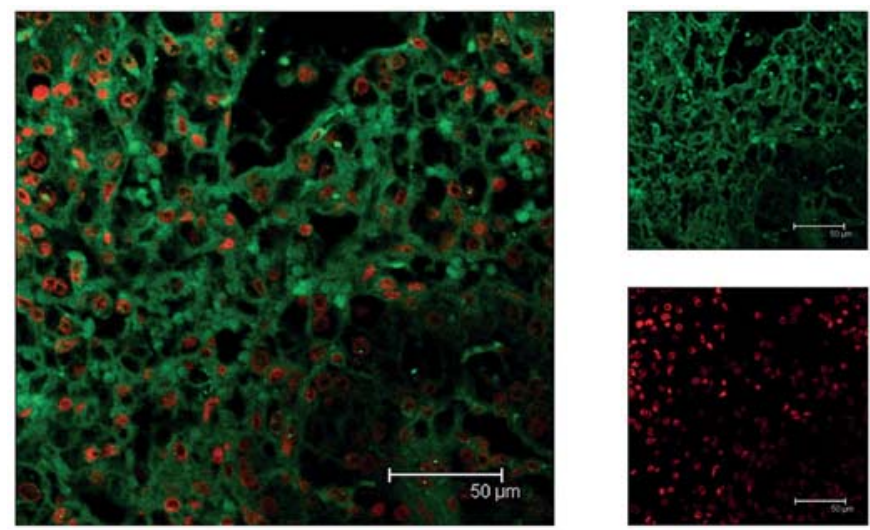

B
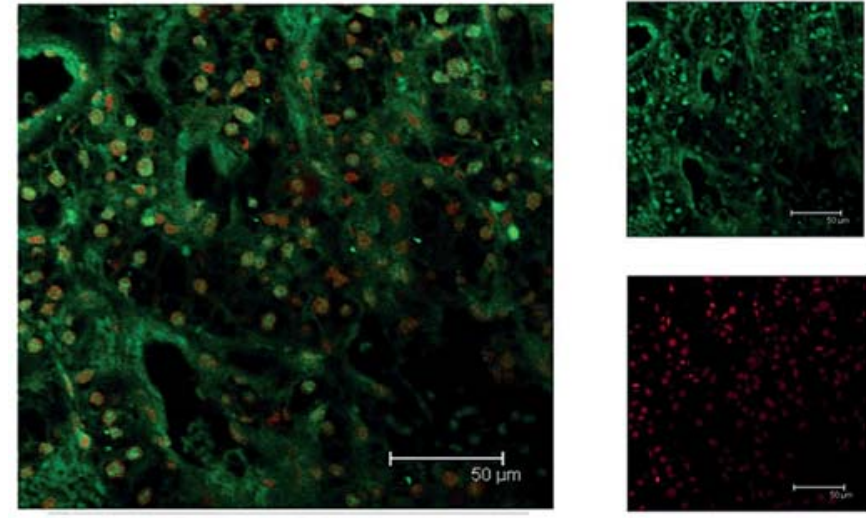

C
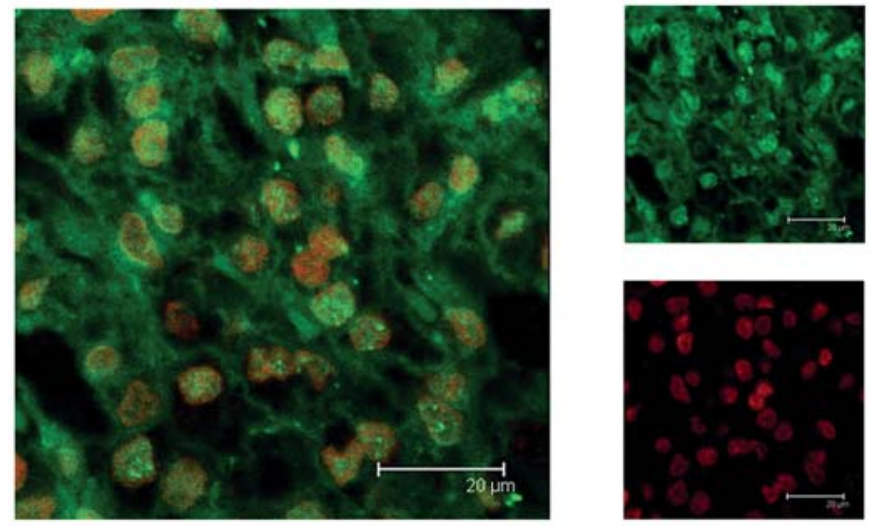

Figure 4. Expression pattern of CXCR4 in primary and metastatic RCC lesions. Histological sections of primary (A) and metastatic (B and C) RCC lesions were immunofluorescently stained for CXCR4 (green) and for nuclei (propidium iodide, red). In the fused images, costaining shows as yellow.

CXCR4 is on the cell surface, the majority of CXCR4 exists in early endosomes, probably recycling to the cell surface (16). CXCR4 internalizes via clathrin-coated pits (27) and its internalization requires $\mathrm{Hsc} 73$ to uncoat the clathrin-coated pits for fusion to the endosome (20).

Upon stimulating cells with $200 \mathrm{nM}(\sim 1600 \mathrm{ng} / \mathrm{ml})$ SDF-1, surface CXCR4 is rapidly depleted from the cell surface within 30-60 min (17). If SDF-1 stimulation is then removed, the cell surface CXCR4 returns to normal levels within $15 \mathrm{~min}$, suggesting that it recycles from the endosomes (17). Stimulation with $10 \mathrm{nM}(\sim 80 \mathrm{ng} / \mathrm{ml})$ SDF-1 causes CXCR4 to 
move from the endosomal compartments to lysosomes within $30 \mathrm{~min}$ (18). Our results show that following stimulation of A-498 cells with $200 \mathrm{ng} / \mathrm{ml} \mathrm{SDF-1,} \mathrm{the} \mathrm{internal} \mathrm{CXCR4} \mathrm{was}$ found in endo/lysosomal compartments between 0-6 h, but at $12 \mathrm{~h}$ CXCR4 was seen exiting the endo/lysosomal vesicles and becoming diffusely distributed throughout the cytoplasm and nucleus and that by $24 \mathrm{~h}$ CXCR 4 was completely diffused throughout the cytoplasm and nucleus. Western blotting detected a small amount of CXCR4 in the nuclear fractions from unstimulated A-498 cells overexpressing CXCR4 and a large amount of CXCR4 in the nuclear fraction after SDF-1 stimulation. This confirmed that the CXCR4 nuclear localization seen by immunofluorescent microscopy was not an artifact. The small amount of CXCR4 found in the nuclear fraction of unstimulated cells could represent some early nuclear translocation of CXCR4 which was undetectable by immunofluorescent microscopy or represent a technical artifact due to either the overexpression of CXCR4 or crosscontamination of the nuclear and cytoplasmic fractions.

This nuclear localization of CXCR4 is comparable to the mechanism observed for the epidermal growth factor receptor (EGFR), another membrane receptor (23). Lin et al demonstrated that when the epidermal growth factor binds to the EGFR, EGFR translocates to the nucleus, binds to the promoter region of the cyclin D1 gene, and increases its transcription (23), leading to increased proliferation. Thus, the nuclear translocation of EGFR precedes its action as a transcriptional regulator causing an observable phenotype. It will be of interest to examine whether CXCR4 plays a similar biological role when it translocates to the nucleus.

In order to see if CXCR4 nuclear translocation might have functional consequences, we performed an invasion ability test. SDF-1 stimulation of CXCR4 is well known to cause cells to traverse a Matrigel-coated membrane $(25,28)$, a test for metastatic phenotype. We repeated this test with A-498 cells that had a short or long period of exposure to SDF-1. The long SDF-1 exposure should have induced full nuclear translocation of CXCR4 and was found to induce significantly more invasion of the Matrigel. This suggests that the nuclear localization of CXCR4 does have functional consequences. CXCR4 could be involved in transcriptional regulation in the nucleus and control genes that regulate invasion, migration and chemoattraction.

The cell culture experiments suggest that CXCR4 has both fast and slow responses to SDF-1 stimulation. Signal transduction pathways connected to $\mathrm{CXCR} 4$, such as $\mathrm{G}$ protein mediated signaling (14), are a fast response. We hypothesize that the nuclear localization of CXCR4 may represent a slow response requiring long periods of stimulation, resulting in a commitment to a migratory or metastatic phenotype only if there is sustained signaling. However, our experiments provide little evidence to support this hypothesis. Future study should examine whether sustained stimulation with SDF-1 or only temporary stimulation with SDF-1 followed by a $24 \mathrm{~h}$ incubation period is necessary for nuclear localization.

$\mathrm{RCC}$ is known to involve CXCR4/SDF-1. Having discovered nuclear localization of CXCF4 and that it influences a metastatic property, invasion through Matrigel, we looked for evidence of CXCR4 nuclear localization in RCC cells. We did indeed find evidence that metastatic RCC cells had nuclear localization of CXCR4, suggesting that the nuclear localization and the metastatic nature of the lesions were related. The distribution of CXCR4 in metastatic RCC cells was exactly the same as that of A-498 cells stimulated with SDF-1, diffuse cytoplasmic and nuclear staining. However, the primary RCC cells were not exactly the same as unstimulated A-498 cells: the RCC cells had diffuse cytoplasmic, nonnuclear staining rather than staining in the endosome/lysosome. Unless this is a technical artifact, diffuse cytoplasmic CXCR4 staining represents a third intracellular distribution pattern for CXCR4 along with endo/lysosomal and nuclear/cytoplasmic staining. Both endo/lysosomal $(16,18)$ and diffuse cytoplasmic (22) CXCR4 staining patterns have been described in the literature, but the implication of the diffuse cytoplasmic pattern has not been assessed. Due to the wide range of conditions used in various studies, it is inconclusive whether diffuse cytoplasmic staining is artifact or is real and meaningful.

Holland et al also found differences in CXCR4 activity between metastatic vs. non-metastatic breast cancer (12), suggesting that our findings may be applicable to other cancer types. Further study will be necessary to unravel the many complexities of this signaling system, but the fact that nuclear staining for CXCR4 could identify metastatic RCC cells may find immediate use in diagnostic immunohistochemistry if the phenomenon is verified.

The SDF-1/CXCR4 axis plays significant roles in tumorigenesis and metastasis (4). CXCR4 is one of the major chemokine receptors on several cancer cells $(1,29-33)$. Increasing lines of evidence support the proposal that the SDF-1/CXCR4 axis plays a role in the proliferation, adhesion, chemotaxis and invasion of several tumors (34-36). Further, silencing of CXCR4 can inhibit the proliferation, adhesion, chemotaxis and invasion of tumor cells (37). The nuclear localization of CXCR4 may play important roles in metastasis of renal cell carcinoma. However, the targets in the nucleus which interact with CXCR4 remain unknown. It would be of interest to determine which domain of CXCR4 is necessary for translocation to nucleus and what is activated after nuclear localization of CXCR4.

\section{Acknowledgements}

The authors would like to thank Professor Cai Zailong and Professor Mao Jifang for expert technical assistance. This study was supported by a grant from the National Natural Science Foundation of China and was partially completed in the Laboratory Center for Medical Science, Changhai Hospital.

\section{References}

1. Pan J, Mestas J, Burdick MD, et al: Stromal derived factor-1 (SDF-1/CXCL12) and CXCR4 in renal cell carcinoma metastasis. Mol Cancer 5: 56, 2006.

2. Muller A, Homey B, Soto H, et al: Involvement of chemokine receptors in breast cancer metastasis. Nature 410: 50-56, 2001.

3. Jones J, Marian D, Weich E, et al: CXCR4 chemokine receptor engagement modifies integrin dependent adhesion of renal carcinoma cells. Exp Cell Res 313: 4051-4065, 2007.

4. Balkwill F: The significance of cancer cell expression of the chemokine receptor CXCR4. Semin Cancer Biol 14: 171-179, 2004.

5. Zlotnik A: Involvement of chemokine receptors in organ-specific metastasis. Contrib Microbiol 13: 191-199, 2006. 
6. Sehgal A, Keener C, Boynton AL, Warrick J and Murphy GP: CXCR-4, a chemokine receptor, is overexpressed in and required for proliferation of glioblastoma tumor cells. J Surg Oncol 69: 99-104, 1998.

7. Koshiba T, Hosotani R, Miyamoto Y, et al: Expression of stromal cell-derived factor 1 and CXCR4 ligand receptor system in pancreatic cancer: a possible role for tumor progression. Clin Cancer Res 6: 3530-3535, 2000.

8. Gerritsen ME, Peale FV and Wu T: Gene expression profiling in silico: relative expression of candidate angiogenesis associated genes in renal cell carcinomas. Exp Nephrol 10: 114-119, 2002.

9. Haviv YS, van Houdt WJ, Lu B, Curiel DT and Zhu ZB: Transcriptional targeting in renal cancer cell lines via the human CXCR4 promoter. Mol Cancer Ther 3: 687-691, 2004.

10. Ding Y, Zhang L, Goodwin JS, et al: Plectin regulates the signaling and trafficking of the HIV-1 co-receptor CXCR4 and plays a role in HIV-1 infection. Exp Cell Res 314: 590-602, 2008.

11. Hidalgo A, Sanz-Rodriguez F, Rodriguez-Fernandez JL, et al: Chemokine stromal cell-derived factor-1alpha modulates VLA-4 integrin-dependent adhesion to fibronectin and VCAM-1 on bone marrow hematopoietic progenitor cells. Exp Hematol 29: 345-355, 2001.

12. Holland JD, Kochetkova M, Akekawatchai C, Dottore M, Lopez A and McColl SR: Differential functional activation of chemokine receptor CXCR4 is mediated by $\mathrm{G}$ proteins in breast cancer cells. Cancer Res 66: 4117-4124, 2006.

13. Kucia M, Jankowski K, Reca R, et al: CXCR4-SDF-1 signalling, locomotion, chemotaxis and adhesion. J Mol Histol 35: 233-245, 2004.

14. Vila-Coro AJ, Rodriguez-Frade JM, Martin De Ana A, Moreno-Ortiz MC, Martinez AC and Mellado M: The chemokine SDF-1alpha triggers CXCR4 receptor dimerization and activates the JAK/STAT pathway. FASEB J 13: 1699-1710, 1999.

15. Wang J, Guan E, Roderiquez G, Calvert V, Alvarez R and Norcross MA: Role of tyrosine phosphorylation in ligandindependent sequestration of CXCR4 in human primary monocytes-macrophages. J Biol Chem 276: 49236-49243, 2001.

16. Zhang Y, Foudi A, Geay JF, et al: Intracellular localization and constitutive endocytosis of CXCR4 in human CD34+ hematopoietic progenitor cells. Stem Cells 22: 1015-1029, 2004

17. Amara A, Gall SL, Schwartz O, et al: HIV coreceptor downregulation as antiviral principle: SDF-1alpha-dependent internalization of the chemokine receptor CXCR4 contributes to inhibition of HIV replication. J Exp Med 186: 139-146, 1997.

18. Tarasova NI, Stauber RH and Michejda CJ: Spontaneous and ligand-induced trafficking of CXC-chemokine receptor 4. J Biol Chem 273: 15883-15886, 1998 .

19. Li R, Luo C, Mines M, Zhang J and Fan GH: Chemokine CXCL12 induces binding of ferritin heavy chain to the chemokine receptor CXCR4, alters CXCR4 signaling, and induces phosphorylation and nuclear translocation of ferritin heavy chain. J Biol Chem 281: 37616-37627, 2006.

20. Ding Y, Li M, Zhang J, et al: The 73-kDa heat shock cognate protein is a CXCR4 binding protein that regulates the receptor endocytosis and the receptor-mediated chemotaxis. Mol Pharmacol 69: 1269-1279, 2006.

21. Rey M, Valenzuela-Fernandez A, Urzainqui A, et al: Myosin IIA is involved in the endocytosis of CXCR4 induced by SDF-1 alpha. J Cell Sci 120: 1126-1133, 2007.
22. Cheng ZJ, Zhao J, Sun Y, et al: Beta-arrestin differentially regulates the chemokine receptor CXCR4-mediated signaling and receptor internalization, and this implicates multiple interaction sites between beta-arrestin and CXCR4. J Biol Chem 275: 2479-2485, 2000.

23. Lin SY, Makino K, Xia W, et al: Nuclear localization of EGF receptor and its potential new role as a transcription factor. Nat Cell Biol 3: 802-808, 2001.

24. Schrader AJ, Lechner O, Templin M, et al: CXCR4/CXCL12 expression and signalling in kidney cancer. Br J Cancer 86: 1250-1256, 2002.

25. Wang J, He L, Combs CA, Roderiquez G and Norcross MA Dimerization of CXCR4 in living malignant cells: control of cell migration by a synthetic peptide that reduces homologous CXCR4 interactions. Mol Cancer Ther 5: 2474-2483, 2006.

26. Nguyen DH and Taub D: CXCR4 function requires membrane cholesterol: implications for HIV infection. J Immunol 168: 4121-4126, 2002

27. Venkatesan S, Rose JJ, Lodge R, Murphy PM and Foley JF: Distinct mechanisms of agonist-induced endocytosis for human chemokine receptors CCR5 and CXCR4. Mol Biol Cell 14: 3305-3324, 2003

28. Ding Z, Issekutz TB, Downey GP and Waddell TK: L-selectin stimulation enhances functional expression of surface CXCR4 in lymphocytes: implications for cellular activation during adhesion and migration. Blood 101: 4245-4252, 2003.

29. Scala S, Giuliano P, Ascierto PA, et al: Human melanoma metastases express functional CXCR4. Clin Cancer Res 12: 2427-2433, 2006

30. Fernandis AZ, Prasad A, Band H, Klosel R and Ganju RK: Regulation of CXCR4-mediated chemotaxis and chemoinvasion of breast cancer cells. Oncogene 23: 157-167, 2004.

31. Yasumoto K, Koizumi K, Kawashima A, et al: Role of the CXCL12/CXCR4 axis in peritoneal carcinomatosis of gastric cancer. Cancer Res 66: 2181-2187, 2006.

32. Zeelenberg IS, Ruuls-Van Stalle L and Roos E: The chemokine receptor CXCR4 is required for outgrowth of colon carcinoma micrometastases. Cancer Res 63: 3833-3839, 2003.

33. Taichman RS, Cooper C, Keller ET, Pienta KJ, Taichman NS and McCauley LK: Use of the stromal cell-derived factor-1/ CXCR4 pathway in prostate cancer metastasis to bone. Cancer Res 62: 1832-1837, 2002.

34. Barbieri F, Bajetto A, Porcile C, et al: CXC receptor and chemokine expression in human meningioma: SDF1/CXCR4 signaling activates ERK1/2 and stimulates meningioma cell proliferation. Ann NY Acad Sci 1090: 332-343, 2006.

35. Wulfaenger J, Niedling S, Riemann D and Seliger B Aminopeptidase N (APN)/CD13-dependent CXCR4 downregulation is associated with diminished cell migration, proliferation and invasion. Mol Membr Biol 25: 72-82, 2008.

36. Wu M, Chen Q, Li D, et al: LRRC4 inhibits human glioblastoma cells proliferation, invasion, and proMMP-2 activation by reducing SDF-1 alpha/CXCR4-mediated ERK1/2 and Akt signaling pathways. J Cell Biochem 103: 245-255, 2008.

37. Wen DS, Zhu XL, Guan SM, Wu YM, Yu LL and Wu JZ: Silencing of CXCR4 inhibits the proliferation, adhesion, chemotaxis and invasion of salivary gland mucoepidermoid carcinoma Mc3 cells in vitro. Oral Oncol 44: 545-554, 2008. 